\title{
A Rare Case of Measles-Associated Hemophagocytic Lymphohistiocytosis in an Infant
}

\author{
Theano Lagousi $^{1}$, Paraskevi Korovessi ${ }^{1}$, Eleni Panagouli ${ }^{1}$, Vasilis Tsagris ${ }^{1}$, Stavroula Kostaridou ${ }^{1}$ \\ 1. Paediatrics, Penteli Children's Hospital, Athens, GRC
}

Corresponding author: Theano Lagousi, theanolagousi@hotmail.com

\begin{abstract}
Measles continues to be a threat in most European countries due to suboptimum vaccination coverage. Although measles leads to several complications, measles-related hemophagocytic lymphohistiocytosis (HLH) has been rarely reported. Herein, we present a case of a four-month-old male infant, the first child of unrelated, healthy parents, with no significant medical history or unexplained infant death in the family, otherwise healthy, who was diagnosed with measles-associated HLH and was successfully treated with IV dexamethasone and IV immunoglobulin (IVIG). Additionally, we review previously reported cases of HLH secondary to measles and highlight the diagnostic and therapeutic challenges associated with its early recognition and treatment. High suspicion, early recognition, and appropriate treatment are essential for a favorable outcome of measles-associated HLH.
\end{abstract}

Categories: Pediatrics, Hematology

Keywords: measles, secondary hemophagocytic lymphohistiocytosis, immunoglobulin, vaccination

\section{Introduction}

A large measles epidemic has affected European countries in the past three years [1]. During this period, Greece saw the second largest measles outbreak in Europe [1]. In 2017, based on the local surveillance data from the Hellenic Center for Disease Control \& Prevention, 968 cases were recorded, after three years without local endemic measles transmission, starting from a cluster of three imported cases in Northern Greece, concerning unvaccinated Romanian Roma siblings [2]. The outbreak has spread all over Greece, reaching 3150 reported cases, primarily in the southwestern country. Most cases were of Roma origin, especially children < 10 -year-old, followed by Greek nationals, mostly young adults, not immune to measles and some nonvaccinated healthcare professionals (HCPs) [2]. Overall, four deaths have been reported. Broad vaccination campaigns that were implemented afterward in refugee/migrant hosting sites prevented the extensive spread of measles in such populations [2]. Currently, further efforts focus on raising awareness among HCPs and on organizing vaccination campaigns in hard-to-reach vulnerable populations such as the Roma population where vaccination rates remain low.

Received 05/04/2020

Review began 05/11/2020 Review ended 05/21/2020 Published 05/23/2020

๑) Copyright 2020

Lagousi et al. This is an open access article distributed under the terms of the Creative Commons Attribution License CC-BY 4.0., which permits unrestricted use, distribution, and reproduction in any medium, provided the original author and source are credited.
Hemophagocytic lymphohistiocytosis (HLH) is a rare and occasionally fatal disorder characterized by abnormal proliferation of macrophages, hypercytokinemia, and T-cell immunosuppression leading to multiorgan failure [3-4].

Hemophagocytic lymphohistiocytosis may be familial, affecting infants (fHLH), or occur at any age secondary to infection, malignancy, or rheumatologic disease (sHLH) [5]. In 2001 the Histocyte Society revised the diagnostic criteria and therapeutic approach for HLH (HLH-2004-protocol) [5]. For the diagnosis, five of the eight criteria [1:fever, 2:splenomegaly, 3:cytopenias at least in two blood cell-lines,

4:hypertriglyceridemia and/or hypofibrinogenemia, 5 :hemophagocytosis in bone marrow, spleen, or lymph nodes, 6:low or absent natural killer (NK)-cell activity, 7 :hyperferritinemia, 8:high levels of soluble-IL2r] must be fulfilled. Other clinical and laboratory findings supporting HLH-diagnosis include cerebrospinalfluid pleocytosis and/or elevated spinal fluid protein, histologically confirmed chronic hepatitis, cerebromeningeal symptoms, lymphadenopathy, jaundice, edema, rash, transaminitis, hypoproteinemia, hyponatremia, hyperbilirubinemia, and serum lactate dehydrogenase (LDH)>1.000 IU/L [5-6].

Although measles is associated with high morbidity and mortality rates due to several complications, measles-induced HLH has been rarely described. Herein, we describe a case of an infant with potentially lifethreatening measles due to associated HLH. Additionally, we review reported cases of measles-associated HLH and highlight the diagnostic and therapeutic challenges associated with its early recognition and treatment.

\section{Case Presentation}

A four-month-old male infant, the first child of unrelated, healthy parents, with no significant medical history or unexplained infant death in the family, otherwise healthy, presented with severe respiratory 


\section{Cureus}

distress due to measles-associated pneumonitis (nine days after the disease onset). His initial physical examination revealed a febrile in poor general condition, with a respiratory rate at $70 / \mathrm{min}$, oxygen saturation at 93\%-94\%, and diffuse crackles and wheezing. He had a generalized erythematous maculopapular rash, while Koplik spots were not visible. There was no conjunctivitis, lymphadenopathy, or hepatosplenomegaly. Admission laboratory-tests including full-blood-count, C-reactive protein (CRP), procalcitonin, kidney/liver function, and serum electrolytes were normal. Measles IgM antibodies were detected serologically. Initial and repeated blood-cultures did not yield any pathogen. Abdominal ultrasound in admission was normal, while chest X-ray presented only mild inflitrations and no signs of pneumonia (Figure 1). He was met on nebulized racemic epinephrine and oxygen administration, and was started on IV cefotaxime due to a potential concomitant bacterial co-infection, suspecting sepsis due to affected clinical condition.

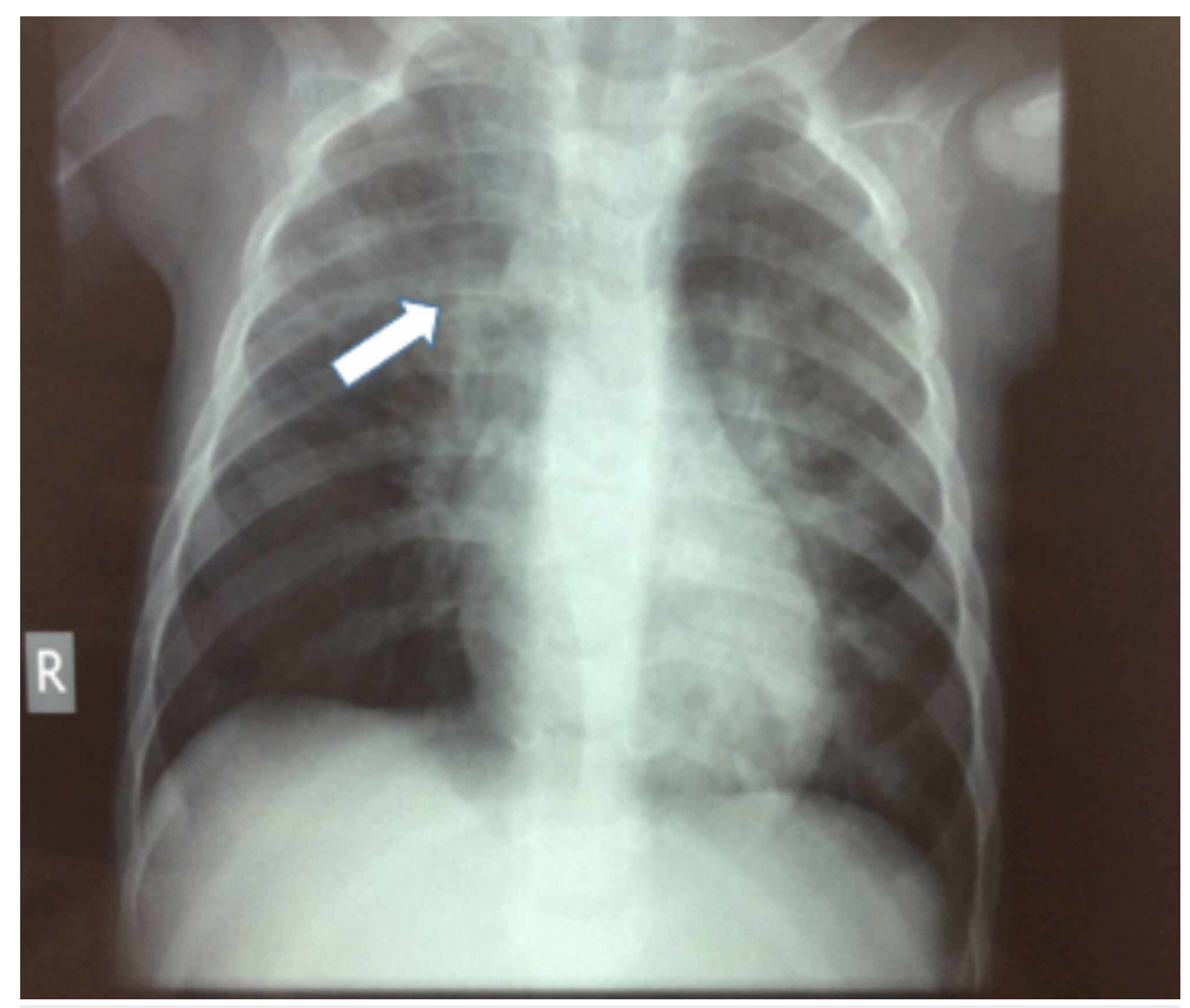

\section{FIGURE 1: Patient's chest X-ray.}

Chest X-ray in admission showing mild infiltration

Five days later, he was still in poor general condition with persistent respiratory distress and fever, while hepatosplenomegaly was revealed in the new ultrasound (Figure 2). 


\section{Cureus}

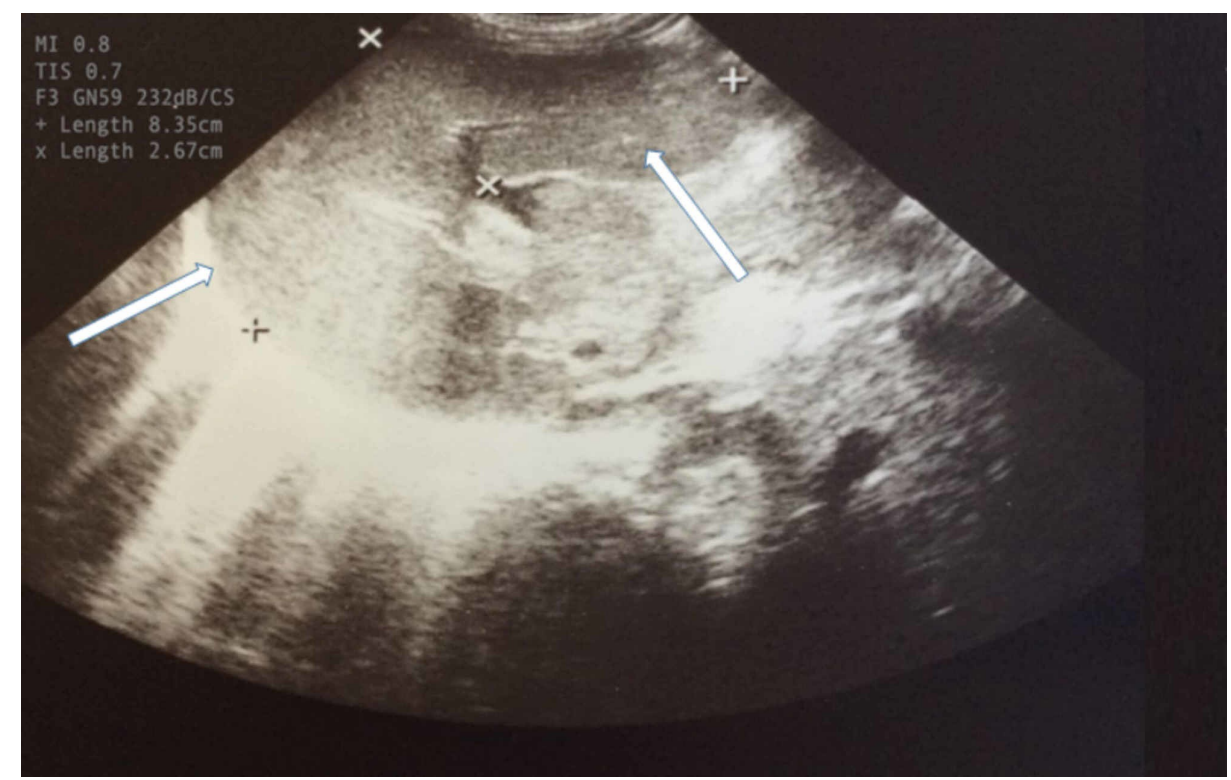

\section{FIGURE 2: Patient's ultrasound.}

The second (repetitive) ultrasound showed splenomegaly $(8.35 \mathrm{~cm})$

Further investigations revealed substantially elevated serum ferritin $(\sim 10000 \mu \mathrm{g} / \mathrm{L})$ and slight

hypofibrinogenemia $(1.34 \mathrm{~g} / \mathrm{L})$. All the biochemical exams performed in the patient are presented in Table 1. As HLH was considered, bone marrow (BM) aspiration was performed, showing hemophagocytosis without blasts (Figure 3). 


\section{Cureus}

\begin{tabular}{|c|c|c|c|c|c|c|c|c|c|c|}
\hline \multirow{2}{*}{ Laboratory tests } & \multicolumn{10}{|c|}{ Hospitalization day } \\
\hline & 2 & 3 & 4 & 5 & 6 & 7 & 8 & 9 & 10 & Normal range \\
\hline WBC $\left(\mathrm{X} 10^{3} / \mathrm{mm}^{3}\right)$ & 16.4 & 20.6 & 11 & 12.5 & 4.09 & 5.72 & 10 & 14.1 & 15.2 & $6-17.5$ \\
\hline Neutrophils $\left(10^{3} / \mathrm{mm} 3\right)$ & 2 & 3.5 & 2.7 & 2.1 & 1.9 & 1.5 & $0.7^{\S}$ & 2.1 & 4.2 & $1-8.5$ \\
\hline $\mathrm{HgB}(\mathrm{g} / \mathrm{dL})$ & 13.6 & 13.1 & 13 & 13.2 & 12 & 11.4 & 10.3 & $8.2^{\S}$ & 9.9 & $9.5-14.1$ \\
\hline PLT $\left(\mathrm{X} 10^{3} / \mathrm{mm}^{3}\right)$ & 372 & 351 & 269 & 252 & 190 & 123 & $85^{\S}$ & 135 & 160 & $150-350$ \\
\hline SGOT (IU/L) & 56 & 103 & 379 & $454^{*}$ & 280 & 204 & 161 & 56 & 49 & $15-60$ \\
\hline SGPT (IU/L) & 33 & 42 & 172 & $216^{\star}$ & 174 & 192 & 271 & 150 & 106 & $13-45$ \\
\hline Serum albumin (g/dL) & & & & 3.7 & 3.1 & 3.3 & & & 3.9 & $3.4-5.4$ \\
\hline Sodium (mEq/L) & 142 & 139 & & 137 & & 140 & & 139 & 135 & $135-145$ \\
\hline Ferritin $(\mu \mathrm{g} / \mathrm{L})$ & & & 7230 & $9943^{\S}$ & 6400 & 4515 & 2538 & 1163 & 600 & $50-200$ \\
\hline TGL (mg/dL) & & & 140 & 168 & 245 & $592^{\S}$ & 575 & 297 & 181 & $30-86$ \\
\hline LDH (IU/L) & & & & $1540^{*}$ & 971 & 519 & 367 & 252 & 242 & $180-430$ \\
\hline APTT (s) & & & & 47.1 & & 30. & 32.4 & 26.5 & & $24-36$ \\
\hline Fibrinogen (g/L) & & & & $1.34^{\S}$ & & 1.9 & & & & $1.7-4.05$ \\
\hline D-Dimers $(\mu \mathrm{g} / \mathrm{mL})$ & & & & $3.5^{\star}$ & & 1.6 & & & & $<0.5$ \\
\hline
\end{tabular}

\section{TABLE 1: Biochemical tests during patient's hospitalization.}

WBC: white blood cells, HgB: hemoglobin, PLT: platelets, APPT: activated partial thromboplastin time, SGOT: serum glutamic oxaloacetic transaminase, SGPT: serum glutamic pyruvic transaminase, LDH: lactate dehydrogenase, TGL: triglycerides.

Normal range is also depicted (last column).

§: laboratory test values fulfilling HLH-2004-criteria. *: supportive HLH-2004-criteria fulfilled when HLH diagnosis was set. 
FIGURE 3: Periodic acid-Schiff staining of the bone marrow aspirate.

The black arrow indicates a macrophage which phagocytized an immature red blood cell

Our patient fulfilled five out of eight criteria of HLH-2004-criteria; fever, splenomegaly, hyperferritinemia, hypofibrinogenemia, and BM hemophagocytosis. Transaminitis (aspartate transaminase $454 \mathrm{IU} / \mathrm{L}$ and alanine transaminase $216 \mathrm{IU} / \mathrm{L})$ and increased D-dimers $(3.5 \mu \mathrm{g} / \mathrm{mL})$ and lactate dehydrogenase (1540 IU/L) further supported HLH-diagnosis. Hypertriglyceridemia (reaching $592 \mathrm{mg} / \mathrm{dL}$ ) and a drop in all blood celllines (reaching the following values: neutrophils 0.7 x 109/L, hemoglobin $82 \mathrm{~g} / \mathrm{L}$, and platelets 85 x 109/L) presented later in the disease course. As there was no diagnostic uncertainty based on the above criteria, NK function or CD25 levels were not measured. The most common triggering causes of sHLH including infectious agents (EBV, CMV, parvovirus B19, enterovirus, adenovirus, influenzae virus, and leishmania) and basic rheumatologic investigations (antinuclear/anti-double stranded DNA antibodies) were all negative. Total subclasses of immunoglobulins and peripheral blood immunophenotype were normal. Therefore, our patient was diagnosed as having measles-associated SHLH and was managed with IV dexamethasone and IV immunoglobulin (IVIG), and supportive chemoprophylaxis. He progressively recovered, staying afebrile from the first week of treatment onset. Dexamethasone was continued for eight weeks and the patient remained well. No signs of relapse occurred 15 months later. The clinical course and the progression of the patient's laboratory parameters are shown in Figure 4.

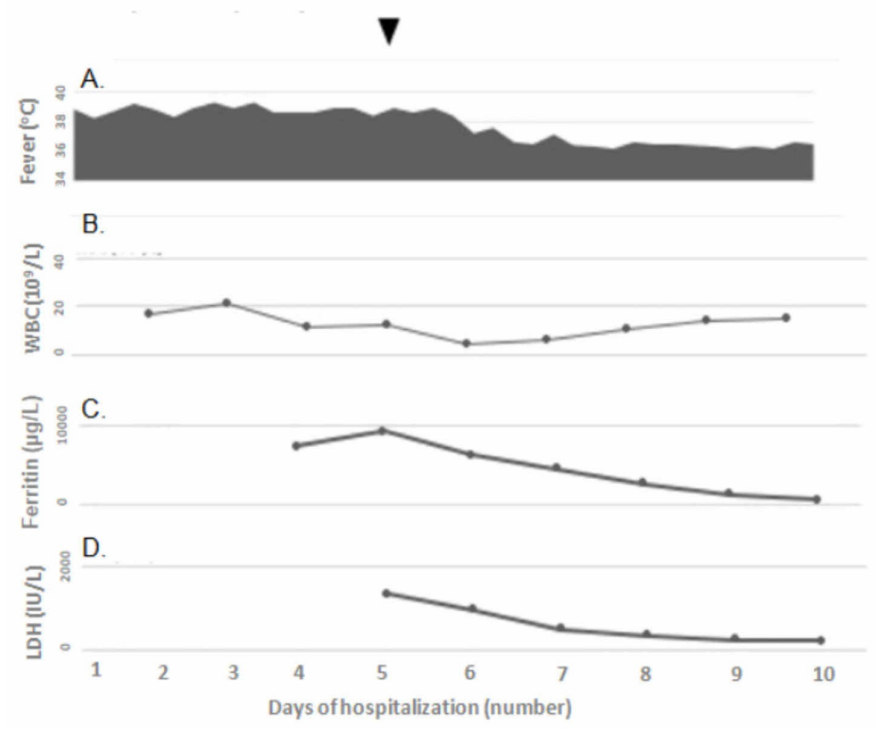

\section{FIGURE 4: The clinical course of the patient.}

The arrow indicates the onset of the treatment with dexamethasone and intravenous immunoglobulin (IVIG). The numbers on the horizontal axis represent the days of hospitalization. A. Body temperature. B,C,D: Laboratory changes: White blood cells (WBC), Ferritin, Lactate dehydrogenase (LDH) respectively. Ferritin and LDH were not asked before Day 4-5 when hemophagocytic lymphohistiocytosis (HLH) was suspected.

\section{Discussion}

While fHLH is caused by mutations in genes involved in perforin-dependent lymphocyte cytotoxicity, sHLH is associated with infection, malignancy, or rheumatic conditions [3-4]. It is often difficult to distinguish the two HLH forms. sHLH is our most likely diagnosis due to the absence of a positive family history, parental consanguinity, and familiar history of unexplained pediatric death. Our patient rapidly responded to the initial treatment with a long remission state, further reducing the possibility of fHLH, that is often more severe, associated with frequent relapses and higher mortality rates. Fever, splenomegaly, hyperferritinemia, cytopenias associated with respiratory distress, and/or multiorgan failure should prompt a strong consideration of sHLH [5]. Remarkably, although blood cell-lines were within the normal range, it is the drop from previous levels that may herald HLH development [5]. fHLH cannot be excluded considering the earlylife onset of HLH as well as previous reports showing that infectious agents may trigger fHLH. Therefore, molecular investigation of fHLH mutations and/or NK-function and soluble-CD25 assessment may be recommended, especially in the context of a possible future reactivation. 
Measles-induced sHLH has been previously reported in five cases (Table 2). All cases presented with pneumonitis [7-11], while only three had also cerebromeningeal symptoms [7, 9]. Three cases occurred in immunocompetent children [8, 10-11]. Among the adults, there was an immunocompetent 18-year-old man with acute respiratory distress [10] and a 17-year-old boy with high risk acute lymphoblastic leukemia [11]. Death occurred in a previously healthy 25 -month-old boy with hemophagocytic syndrome, and acute disseminated demyelinating encephalitis and a 17-year-old boy with high-risk acute lymphoblastic leukemia $[9,11]$. To our knowledge, this is the first case of measles-associated sHLH in an infant.

\begin{tabular}{|c|c|c|c|c|c|}
\hline Reference & Yamamoto et al. [7] & Joshi et al. [8] & Pearl et al. [9] & $\begin{array}{l}\text { Komatsuda et al. } \\
{[10]}\end{array}$ & Huang et al. [11] \\
\hline Gender & Male & Female & Male & Male & Male \\
\hline Age (years) & 8 & School-aged girl & 2 & 18 & 17 \\
\hline Immunocompromised & No & No & No & no & $\begin{array}{l}\text { Yes (acute lymphoblastic } \\
\text { leukemia) }\end{array}$ \\
\hline Treatment & $\begin{array}{l}\text { Cyclosporine A- } \\
\text { methylprednisolone }\end{array}$ & $\begin{array}{l}\text { Supportive } \\
\text { treatment }\end{array}$ & $\begin{array}{l}\text { VP-16, an } \\
\text { epipodophyllotoxin }\end{array}$ & Methylprednisolone & $\begin{array}{l}\text { Steroids/etoposide/plasma } \\
\text { exchange }\end{array}$ \\
\hline Clinical outcome & Favorable & Favorable & Died & Favorable & Died \\
\hline
\end{tabular}

The molecular mechanism involved in measles-induced HLH is not yet defined. CD150/signalinglymphocytic-activation-molecule, a T/B/dendritic cell glycoprotein, is a costimulatory receptor involved in T-cell activation and a cellular receptor for measles virus [12-13]. Considering that CD150-induced signal transduction is controlled by SAP/SH2D1A gene that is aberrant in X-linked lymphoproliferative disease and $\mathrm{fHLH}$, measles virus may affect CD150 function in a way similar to genetic disorder leading to transient excessive macrophages, NK cells and lymphocytes activation, leading in turn to excessive immunoactivation, characterizing sHLH. Therefore, in cases of measles-induced HLH, where a genetic disorder is suspected, SAP/SH2D1A mutation should be investigated; albeit, further research is required to verify this assumption.

Finally, another issue worth mentioning is that measles-induced sHLH management was quite challenging. Firstly, the use of steroids in viral-induced sHLH has been widely debatable. Although, most studies reported no impact on the course of disease progression, a single one described reversal of cytopenia and respiratory failure with pulsed steroids [10]. Therefore, we opted to initiate treatment with immunomodulatory agents (high-dose corticosteroids and IVIG) rather than the standard HLH-2004-protocol, to balance immunosuppression, ready to immediately switch to HLH-2004-protocol at the first sign of failure, something that was not required due to our patient's acute response. This is in accordance with the previous reports supporting the use of IVIG and/or corticosteroids for the first-line treatment of virus-induced sHLH [14-15]. Such an approach may avoid etoposide-induced toxicity. HLH-2004-protocol may be considered in cases with poor clinical response or if disease progression is suspected. Besides, the distinction between sepsis and HLH is often difficult, being two acute hyperinflammatory reactions, potentially fatal without early recognition and with completely different management that is critical for a favorable outcome. A high index of clinical suspicion of measles-associated sHLH is required for early diagnosis and prompt treatment. Sen et al. had suggested that a serum ferritin $>10000 \mu \mathrm{g} / \mathrm{L}$ is strongly supportive of HLH [16]. Similarly, Machowicz et al. suggested that in the presence of hyperferritinemia, splenomegaly, marked cytopenias, hypofibrinogenemia, low CRP, characteristic cytokine profile, hypertriglyceridemia, the other HLH criteria should be assessed [17].

\section{Conclusions}

Amidst a period of measles outbreaks across Europe due to suboptimum vaccination coverage, increased awareness of possible measles-induced sHLH, together with early recognition and initiation of appropriate treatment is crucial to prevent a cytokine storm progressing to multiorgan failure. More intensive efforts are essential to improve vaccination rates and control outbreaks leading to enhanced herd immunity. Such an approach is critical for the protection of unvaccinated young infants as well as immunocompromised patients who are at the highest risk of measles-related sHLH.

\section{Additional Information}

Disclosures 
Human subjects: Consent was obtained by all participants in this study. Conflicts of interest: In compliance with the ICMJE uniform disclosure form, all authors declare the following: Payment/services info: All authors have declared that no financial support was received from any organization for the submitted work. Financial relationships: All authors have declared that they have no financial relationships at present or within the previous three years with any organizations that might have an interest in the submitted work. Other relationships: All authors have declared that there are no other relationships or activities that could appear to have influenced the submitted work.

\section{References}

1. European Centre for Disease Prevention and Control (ECDC): Communicable Diseases Threats Report. Week 35, 27 August-2 September. ECDC, Stockholm; 2017.

2. Georgakopoulou T, Horefti E, Vernardaki A, et al.: Ongoing measles outbreak in Greece related to the recent European-wide epidemic. Epidemiol Infect. 2018, 146:1692-1698. 10.1017/\$0950268818002170

3. Henter JI, Arico M, Elinder G, et al.: Familial hemophagocytic lymphohistiocytosis (primary HLH) . Hematol Oncol Clin North Am. 1998, 12:417-433. 10.1016/s0889-8588(05)70520-7

4. Janka G, Imashuku S, Elinder G, Schneider M, Henter JI: Infection- and malignancy- associated hemophagocytic syndromes: secondary hemophagocytic lymphohistiocytosis. Hematol Oncol Clin North Am. 1998, 12:435-444. 10.1016/s0889-8588(05)70521-9

5. Henter JI, Horne A, Aricó M, et al.: HLH 2004: diagnostic and therapeutic guidelines for hemophagocytic lymphohistiocytosis. Pediatr Blood Cancer. 2007, 48:124-131. 10.1002/pbc.21039

6. Iaria C, Leonardi MS, Buda A, Toro ML, Cascio A: Measles and secondary hemophagocytic lymphohistiocytosis. Emerg Infect Dis. 2012, 18:1529-1530. 10.3201/eid1809.120235

7. Yamamoto K, Otake M, Takayanagi M: Therapeutic effect of cyclosporine: a combined with methylprednisolone pulse therapy on hemophagocytic syndrome with the central nervous system involvement. No To Hattatsu. 2002, 34:66-71.

8. Joshi R, Phatarpekar A, Currimbhoy Z, Desai M: Haemophagocytic lymphohistiocytosis: a case series from Mumbai. Ann Trop Paediatr. 2011, 31:135-140. 10.1179/1465328111Y.0000000009

9. Pearl PL, Abu-Farsakh H, Starke JR, Dreyer Z, Louis PT, Kirkpatrick JB: Neuropathology of two fatal cases of measles in the 1988-1989 Houston epidemic. Pediatr Neurol. 1990, 6:126-130. 10.1016/08878994(90)90046-4

10. Komatsuda A, Chubachi A, Miura AB: Virus-associated hemophagocytic syndrome due to measles accompanied by acute respiratoryfailure. Intern Med. 1995, 34:203-206. 10.2169/internalmedicine.34.203

11. Huang JG, Yeoh AE, Tambyah PA, Suhaila MI: The conundrum in the diagnosis and management of atypical fulminant measles in a leukemia survivor on maintenance chemotherapy. J Pediatr Hematol Oncol. 2017, 39:36. 10.1097/MPH.0000000000000620

12. Howie D, Simarro M, Sayos J, Guirado M, Sancho J, Terhorst C: Molecular dissection of the signaling and costimulatory functions of CD150 (SLAM): CD150/SAP binding and CD150-mediated costimulation. Blood. 2002, 99:957-965. 10.1182/blood.v99.3.957

13. Rota PA, Moss WJ, Takeda M, et al.: Measles. Nat Rev Dis Primers. 2016, 2:16049. 10.1038/nrdp.2016.49

14. Rajajee S, Ashok I, Manwani N, et al.: Profile of hemophagocytic lymphohistiocytosis; efficacy of intravenous immunoglobulin therapy. Indian J Pediatr. 2014, 81:1337-1341. 10.1007/s12098-014-1461-0

15. Buda P, Gietka P, Książyk JB, Machaczka M: The influence of various therapeutic regimens on early clinical and laboratory response and outcome of children with secondary hemophagocytic lymphohistiocytosis. Arch Med Sci. 2018, 14:138-150. 10.5114/aoms.2015.56325

16. Sen ES, Steward CG, Ramanan AV: Diagnosing haemophagocytic syndrome. Arch Dis Child. 2017, 102:279284. 10.1136/archdischild-2016-310772

17. Machowicz R, Janka G, Wiktor-Jedrzejczak W: Similar but not the same: differential diagnosis of HLH and sepsis. Crit Rev Oncol Hematol. 2017, 114:1-12. 10.1016/j.critrevonc.2017.03.023 